CRYSTALLOGRAPHIC COMMUNICATIONS

ISSN 2056-9890

Received 9 February 2016

Accepted 26 February 2016

Edited by H. Stoeckli-Evans, University of Neuchâtel, Switzerland

Keywords: crystal structure; furanocoumarin; oroselone; Artemisia reticulata; photobiological property; hydrogen bonding.

CCDC reference: 1422810

Supporting information: this article has supporting information at journals.iucr.org/e

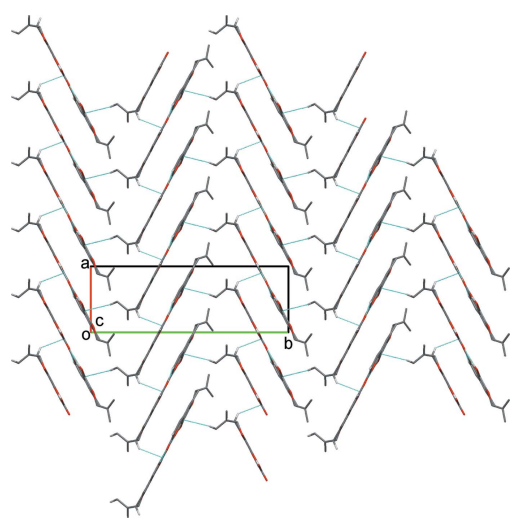

OPEN $\odot$ ACCESS

\section{Crystal structure of a photobiologically active furanocoumarin from Artemisia reticulata}

\author{
A. K. Bauri, ${ }^{a}$ Sabine Foro ${ }^{b}$ and Nhu Quynh Nguyen Do ${ }^{\mathrm{c} *}$
}

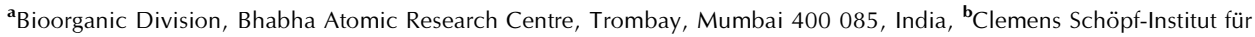
Organische Chemie und Biochemie, Technische Universität Darmstadt, Petersenstrasse 22, D-64287 Darmstadt, Germany, and ${ }^{\mathbf{C}}$ Accident \& Emergency Department, Franco, Vietnamese Hospital, 7-Nguyen, Luong Bang Street, HoChiMinh City, Vietnam. *Correspondence e-mail: nguyendonhuquynh@yahoo.com

The title furanocoumarin, $\mathrm{C}_{14} \mathrm{H}_{12} \mathrm{O}_{4}$ [systematic name: 9-hydroxy-2-(prop-1-en2-yl)-2,3-dihydro-7 $\mathrm{H}$-furo[3,2-g]chromen-7-one], crystallizes with two independent molecules $(A$ and $B)$ in the asymmetric unit. The two molecules differ essentially in the orientation of the propenyl group with respect to the mean plane of the furanocoumarin moiety; the $\mathrm{O}-\mathrm{C}(\mathrm{H})-\mathrm{C}=\mathrm{C}$ torsion angle is 122.2 (7) ${ }^{\circ}$ in molecule $A$ and $-10.8(11)^{\circ}$ in molecule $B$. In the crystal, the $A$ and $B$ molecules are linked via $\mathrm{O}-\mathrm{H} \cdots \mathrm{O}$ hydrogen bonds, forming zigzag $-A-B-$ $A-B$ - chains propagating along [001]. The chains are reinforced by bifurcated $\mathrm{C}-\mathrm{H} \cdots(\mathrm{O}, \mathrm{O})$ hydrogen bonds, forming ribbons which are linked via $\mathrm{C}-\mathrm{H} \cdots \pi$ and $\pi-\pi$ interactions [intercentroid distance $=3.602(2) \AA$ ], forming a threedimensional structure.

\section{Chemical context}

The title furanocoumarin was isolated from the Indian herb $A$. reticulata, by column chromatography over silica gel with a mixture of binary solvent hexane and ethyl acetate by gradient elution. Furanocoumarins, such as oroselone [systematic name: $\quad 8$-(prop-1-en-2-yl)-2H-furo[2,3- $h$ ]chromen-2-one], whose atomic connectivity has been established by spectrometric and spectroscopic analyses (Schroeder et al., 1959; Dorofeenko et al., 1973) but not yet by single crystal X-ray diffraction, exhibit photobiological activity. For example such compounds are employed as photoprotective agents to prevent absorption of harmful UV radiation (Chen et al., 2007, 2009). Anti-oxidant and anti-inflammatory activities have also been reported for furano as well as pyrano coumarins and their derivatives (Appendino et al., 2004; Scott et al., 1976).

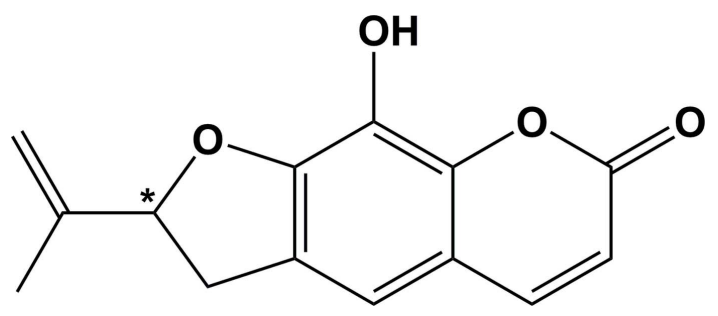

\section{Structural commentary}

The title compound, Fig. 1, crystallizes with two independent molecules $(A$ and $B)$ in the asymmetric unit. The compound is composed of three fused rings (furan, benzene and pyrone) 
Table 1

Selected geometric parameters $\left(\AA{ }^{\circ}\right)$.

\begin{tabular}{lclc}
\hline $\mathrm{C} 2-\mathrm{C} 12$ & $1.500(8)$ & $\mathrm{C} 16-\mathrm{C} 26$ & $1.489(8)$ \\
$\mathrm{C} 12-\mathrm{C} 14$ & $1.313(10)$ & $\mathrm{C} 26-\mathrm{C} 28$ & $1.363(13)$ \\
$\mathrm{C} 12-\mathrm{C} 13$ & $1.461(10)$ & $\mathrm{C} 26-\mathrm{C} 27$ & $1.422(10)$ \\
& & & \\
$\mathrm{C} 14-\mathrm{C} 12-\mathrm{C} 13$ & $122.7(7)$ & $\mathrm{C} 28-\mathrm{C} 26-\mathrm{C} 27$ & $123.5(7)$ \\
$\mathrm{C} 14-\mathrm{C} 12-\mathrm{C} 2$ & $118.9(7)$ & $\mathrm{C} 28-\mathrm{C} 26-\mathrm{C} 16$ & $121.9(6)$ \\
$\mathrm{C} 13-\mathrm{C} 12-\mathrm{C} 2$ & $118.4(5)$ & $\mathrm{C} 27-\mathrm{C} 26-\mathrm{C} 16$ & $114.7(6)$ \\
\hline
\end{tabular}

with hydroxyl and propenyl substituents at positions 9 and 2 , respectively. The furanocoumarin moieties are essentially planar with r.m.s. deviations of $0.05 \AA$ for molecule $A(\mathrm{O} 1 / \mathrm{O} 2 /$ $\mathrm{C} 1-\mathrm{C} 11)$ and $0.079 \AA$ for molecule $B(\mathrm{O} 5 / \mathrm{O} 6 / \mathrm{C} 16-\mathrm{C} 25)$. The furan ring in molecule $A$ has an envelope conformation with atom $\mathrm{C} 2$ as the flap, deviating by 0.120 (4) $\AA$ from the mean plane of the furanocoumarin moiety. In molecule $B$, the furan ring has a twisted conformation on bond $\mathrm{C} 17-\mathrm{C} 16$ with atoms C16 and C17 deviating by $-0.232(6)$ and $0.076(6) \AA$, respectively, from the other atoms of the twisted fivemembered ring. The two molecules differ essentially in the orientation of the propenyl group with respect to the mean plane of the furanocoumarin moiety, as shown by AutoMolFit analysis (Spek, 2009); see Fig. 2. The $\mathrm{O} 1-\mathrm{C} 2-\mathrm{C} 12=\mathrm{C} 14$ torsion angle is $122.2(7)^{\circ}$ in molecule $A$, while the $\mathrm{O} 5-\mathrm{C} 16-$ $\mathrm{C} 26=\mathrm{C} 28$ torsion angle is $-10.8(11){ }^{\circ}$ in molecule $B$. The bond distances and bond angles in the propenyl side chains (C2,C12-C14 in molecule $A$ and $\mathrm{C} 16, \mathrm{C} 26-\mathrm{C} 28$ in molecule $B$ ) also differ in the two molecules (Table 1), probably due to libration and bond rotation. Overall the bond distances and bond angles in the furanocoumarin moieties are in good agreement with the corresponding values reported for related structures (Stemple \& Watson, 1972; Gupta et al., 1993; Singh



Figure 2

The molecular fit (Spek, 2009) of molecules $A$ (black) and $B$ (red) of the title compound.

et al. 1995; Magotra et al., 1995; Thailambal et al., 1986; Thailambal \& Pattabhi, 1987, 1985).

The absolute structure of the molecule in the crystal could not be determined by resonant scattering. In order to determine the chirality at atom $\mathrm{C} 2$ (in molecule $A$; $\mathrm{C} 16$ in molecule $B)$, the circular dichroism (CD) spectrum was measured in a solution of chloroform at concentration of $1 \mathrm{mg} / \mathrm{ml}$ using a cell with path length $1 \mathrm{~cm}$. This $\mathrm{CD}$ measurement revealed that the absolute configuration of atom $\mathrm{C} 2$ (in molecule $A ; \mathrm{C} 16$ in molecule $B$ ) is $S$.

\section{Supramolecular features}

In the crystal, the $A$ and $B$ molecules are linked via $\mathrm{O}-\mathrm{H} \cdots \mathrm{O}$ hydrogen bonds, forming zigzag $-A-B-A-B-$ chains propagating along the $c$-axis direction; see Table 2 and Fig. 3. The chains are reinforced by bifurcated $\mathrm{C}-\mathrm{H} \cdots(\mathrm{O}, \mathrm{O})$ hydrogen

Figure 1

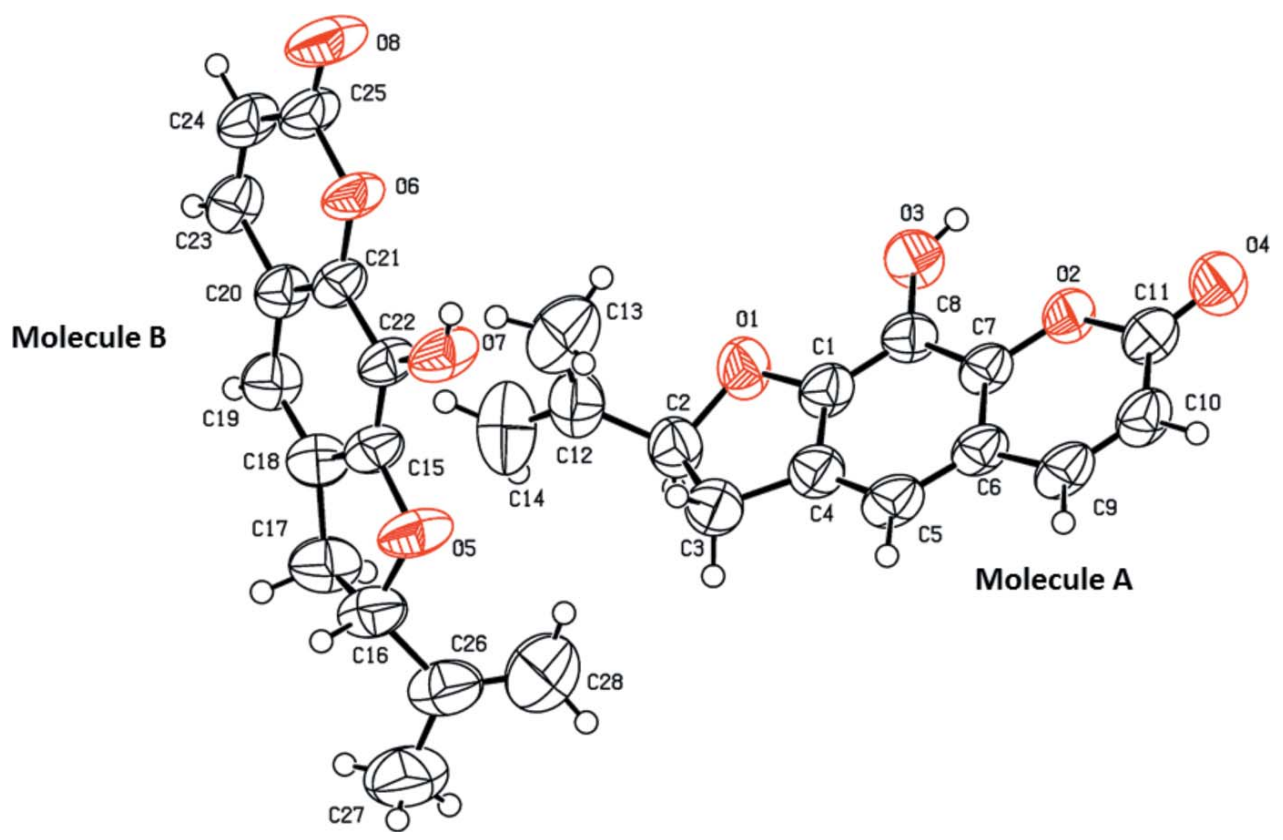

The molecular structure of the two independent molecules $(A$ and $B)$ of the title compound, showing the atom labelling. Displacement ellipsoids are drawn at the $50 \%$ probability level. 
Table 2

Hydrogen-bond geometry $\left(\AA,^{\circ}\right)$.

$\mathrm{Cg} 2$ and $\mathrm{Cg} 9$ are the centroids of rings $\mathrm{O} 2 / \mathrm{C} 6 / \mathrm{C} 7 / \mathrm{C} 9-\mathrm{C} 11$ and $\mathrm{C} 15-\mathrm{C} 22$, respectively.

\begin{tabular}{lllll}
\hline$D-\mathrm{H} \cdots A$ & $D-\mathrm{H}$ & $\mathrm{H} \cdots A$ & $D \cdots A$ & $D-\mathrm{H} \cdots A$ \\
\hline $\mathrm{O} 3-\mathrm{H} 3 O \cdots \mathrm{O} 8^{\mathrm{i}}$ & 0.83 & 1.85 & $2.676(5)$ & 174 \\
$\mathrm{O} 7-\mathrm{H} 7 O \cdots 4^{\text {ii }}$ & 0.84 & 1.85 & $2.671(5)$ & 168 \\
$\mathrm{C} 10-\mathrm{H} 10 \cdots 3^{\text {iii }}$ & 0.93 & 2.53 & $3.199(5)$ & 129 \\
$\mathrm{C} 10-\mathrm{H} 10 \cdots 8^{\text {iv }}$ & 0.93 & 2.50 & $3.415(6)$ & 166 \\
$\mathrm{C} 24-\mathrm{H} 24 \cdots 7^{\mathrm{v}}$ & 0.93 & 2.58 & $3.229(5)$ & 128 \\
$\mathrm{C} 24-\mathrm{H} 24 \cdots \mathrm{O} 4^{\text {vi }}$ & 0.93 & 2.53 & $3.434(5)$ & 164 \\
$\mathrm{C} 3-\mathrm{H} 3 B \cdots C g 2^{\text {vii }}$ & 0.97 & 2.95 & $3.871(5)$ & 160 \\
$\mathrm{C} 13-\mathrm{H} 13 B \cdots C g 9$ & 0.96 & 2.92 & $3.680(9)$ & 137
\end{tabular}

Symmetry codes: (i) $-x+2, y+\frac{1}{2},-z+1$; (ii) $-x+2, y-\frac{1}{2},-z$; (iii) $x, y, z-1$; (iv) $-x+2, y+\frac{1}{2},-z$; (v) $x, y, z+1$; (vi) $-x+2, y-\frac{1}{2},-z+1$; (vii) $x-1, y, z$.

bonds, forming ribbons (Table 2 and Fig. 3). The ribbons are arranged in a herringbone fashion, and are linked via $\mathrm{C}-$ $\mathrm{H} \cdots \pi$ and slipped parallel $\pi-\pi$ interactions, forming a threedimensional network; see Fig. 4 and Table $2\left[C g 2 \cdots C g 9^{\mathrm{i}}=\right.$

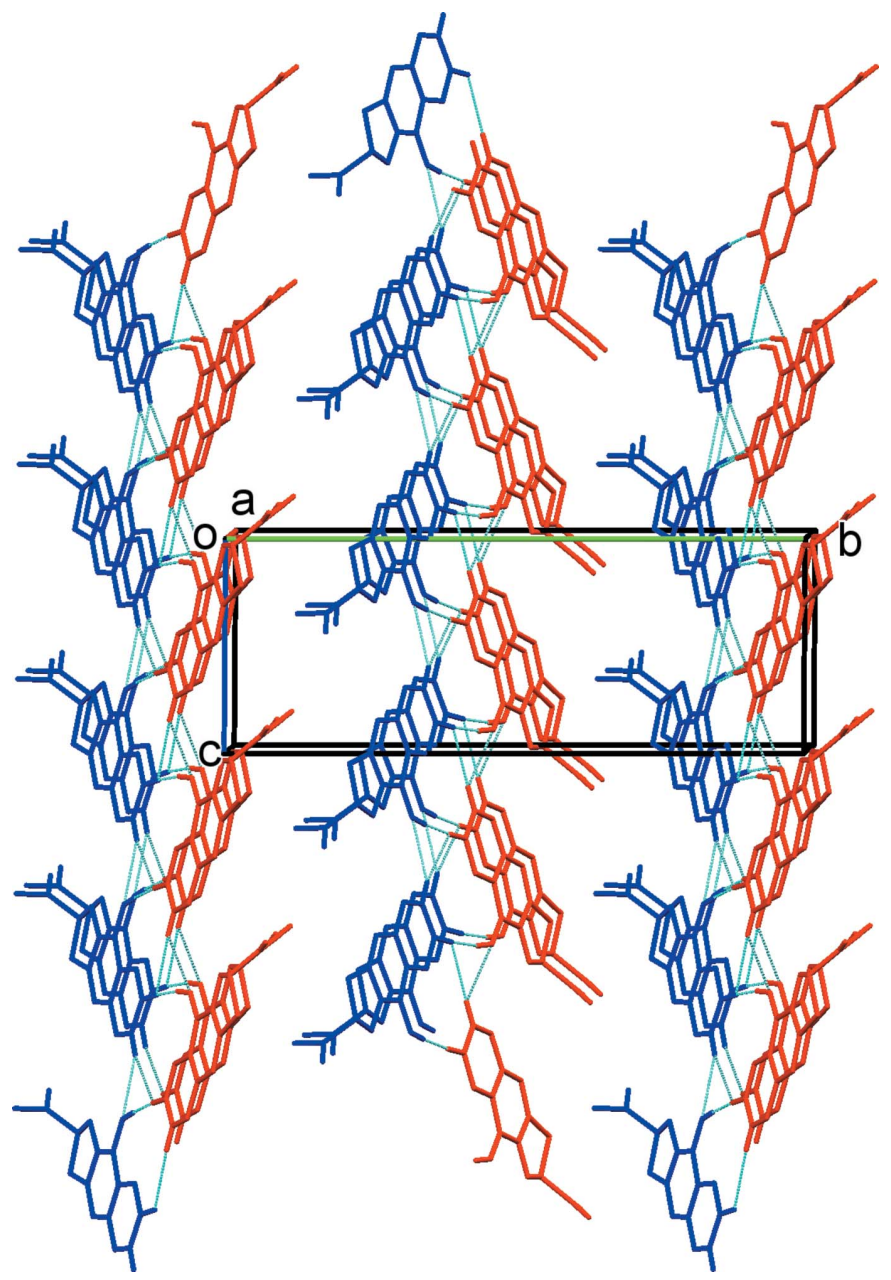

Figure 3

A view along the $a$ axis of the crystal packing of the title compound ( $A$ molecules are blue; $B$ molecules are red). The hydrogen bonds are shown as dashed lines (see Table 2), and C-bound $\mathrm{H}$ atoms not involved in hydrogen bonding have been omitted for clarity.

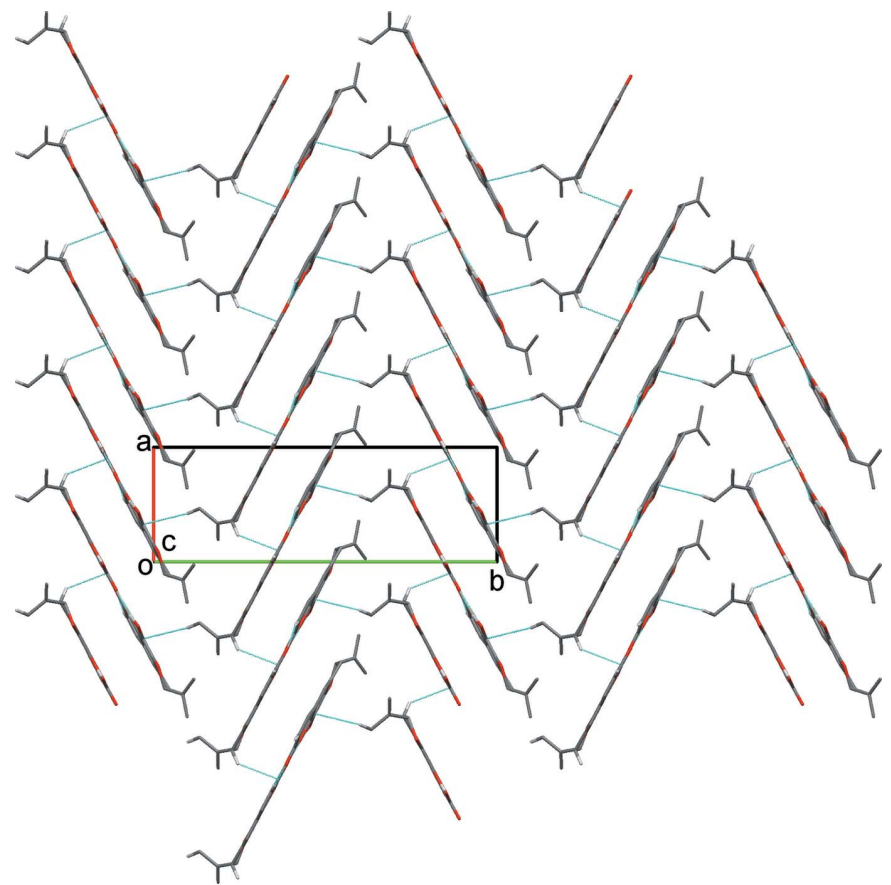

Figure 4

A view along the $c$ axis of the crystal packing of the title compound. Hydrogen bonds and $\mathrm{C}-\mathrm{H} \cdots \pi$ interactions are shown as dashed lines (see Table 2), and $\mathrm{C}$-bound $\mathrm{H}$ atoms not involved in hydrogen bonding have been omitted for clarity.

3.602 (2) $\AA$, interplanar distance $=3.4168$ (2) $\mathrm{A}$, slippage $1.284 \AA$, where $C g 2$ and $C g 9$ are the centroids of rings $\mathrm{C} 1 / \mathrm{C} 4-$ $\mathrm{C} 8$ and $\mathrm{C} 15 / \mathrm{C} 18-\mathrm{C} 22$, respectively; symmetry code: (i) $-x+1$, $\left.y+\frac{1}{2},-z\right]$.

\section{Database survey}

A search of the Cambridge Structural Database (Version 5.37, update November 2015; Groom \& Allen, 2014) gave 21 hits for the furanocoumarin substructure, but only one hit for a 9-hydroxy furanocoumarin, viz. 2,3-dihydro-9-hydroxy-2-(1hydroxy-1-methylethyl)-7H-furo(3,2-g)(1) benzopyran-7-one monohydrate (refcode FUGVOS; Thailambal \& Pattabhi, 1987).

\section{Synthesis and crystallization}

The title compound was isolated as a colourless solid from the methanol extract of A. reticulata by means of column chromatography over silica gel by gradient elution with a mixture of binary solvents system hexane and ethyl acetate. It was purified by reverse-phase high-pressure liquid chromatography. Colourless rod-like crystals suitable for $\mathrm{X}$ ray diffraction analysis were obtained after the title compound was recrystallized three times from ethyl acetate:hexane (1:4) at room temperature by slow evaporation of the solvents (m.p. $498 \mathrm{~K}) .{ }^{1} \mathrm{H}$ NMR data $\left(\mathrm{CHCl}_{3}, 200 \mathrm{MHz}\right) 7.60(d, 1 \mathrm{H}, J=$ $9.6 \mathrm{~Hz}, \mathrm{H}-9), 6.85(s, 1 \mathrm{H}, \mathrm{H}-5), 6.20(d, 1 \mathrm{H}, J=9.6 \mathrm{~Hz}, \mathrm{H}-10)$, $5.35(d d, 1 \mathrm{H}, J=8.8$ and $8.8 \mathrm{~Hz}, \mathrm{H}-7), 5.11\left(s, 1 \mathrm{H}, \mathrm{H}_{\mathrm{a}}-14\right), 4.94$ 
$\left(s, 1 \mathrm{H}, \mathrm{H}_{\mathrm{b}}-14\right), 3.47-3.34(d d, 1 \mathrm{H}, J=9.0$ and $1.2 \mathrm{~Hz}$, $\left.\mathrm{H}_{\mathrm{a}}-3\right), 3.16-3.04\left(d d, 1 \mathrm{H}, J=9.0\right.$ and $\left.1.2 \mathrm{~Hz}, \mathrm{H}_{\mathrm{b}}-3\right), 1.78(\mathrm{~s}, 3 \mathrm{H}$, $-\mathrm{CH}_{3}$ ). EIMS (70 ev) data: $m / z$ (\%) 244(15.9) $\left[M^{+}\right], 226(68.6)$ [ $\left.M^{+}-\mathrm{H}_{2} \mathrm{O}\right), 198$ (100) [base peak], 185 (30),171 (16.8), 155 (30.1), 140 (16.4), 127 (13.5), 115 (25.10,85 (11.1), 75 (22.3), 63 (26.5), 41 (16.0).

\section{Refinement}

Crystal data, data collection and structure refinement details are summarized in Table 3. The hydroxyl $\mathrm{H}$ atoms were located in a difference Fourier map and refined as riding with $U_{\text {iso }}(\mathrm{H})=1.2 U_{\text {eq }}(\mathrm{O})$. The C-bound $\mathrm{H}$ atoms were included in calculated positions and treated as riding atoms: $\mathrm{C}-\mathrm{H}=0.93-$ $0.98 \AA$ with $U_{\text {iso }}(\mathrm{H})=1.2 U_{\text {eq }}(\mathrm{C})$. The limited number of Friedel pairs measured were merged for refinement.

\section{Acknowledgements}

The authors thank Professor Dr Hartmut Fuess, FG Strukturforschung, FB Material und Geowissenschaften, Technische Universität Darmstadt, Petersenstrasse 23, 64287 Darmstadt, for diffractometer time, and Professor N. Komatsu, Shiga University of Medical Science, Shiga, Otsu, Japan, for recording the CD spectrum.

\section{References}

Appendino, G., Bianchi, F., Bader, A., Campagnuolo, C., Fattorusso, E., Taglialatela-Scafati, O., Blanco-Molina, M., Macho, A., Fiebich, B. L., Bremner, P., Heinrich, M., Ballero, M. \& Muñoz, E. (2004). J. Nat. Prod. 67, 532-536.

Chen, Y., Fan, G., Zhang, Q., Wu, H. \& Wu, Y. (2007). J. Pharm. Biomed. Anal. 43, 926-936.

Chen, D., Wang, J., Jiang, Y., Zhou, T., Fan, G. \& Wu, Y. (2009). J. Pharm. Biomed. Anal. 50, 695-702.

Dorofeenko, G. N., Tkachenko, V. V. \& Mezheritskii, V. V. (1973). Chem. Heterocycl. Compd. 9, 948-950.

Enraf-Nonius (1996). CAD-4-PC. Enraf-Nonius, Delft, The Netherlands.

Groom, C. R. \& Allen, F. H. (2014). Angew. Chem. Int. Ed. 53, 662671.

Gupta, V. K., Rajnikant, Goswami, K. N., Mazumdar, S. K., Gupta, B. D. \& Banerjee, S. K. (1993). Cryst. Res. Technol. 28, 187-191.

Macrae, C. F., Bruno, I. J., Chisholm, J. A., Edgington, P. R., McCabe, P., Pidcock, E., Rodriguez-Monge, L., Taylor, R., van de Streek, J. \& Wood, P. A. (2008). J. Appl. Cryst. 41, 466-470.
Table 3

Experimental details.

\begin{tabular}{ll}
\hline Crystal data & \\
Chemical formula & $\mathrm{C}_{14} \mathrm{H}_{12} \mathrm{O}_{4}$ \\
$M_{\mathrm{r}}$ & 244.24 \\
Crystal system, space group & Monoclinic, $P 2_{1}$ \\
Temperature (K) & 299 \\
$a, b, c(\AA)$ & $7.2738(9), 21.426(2), 8.0152(9)$ \\
$\beta\left({ }^{\circ}\right.$ & $100.88(1)$ \\
$V\left(\AA^{3}\right)$ & $1226.7(2)$ \\
$Z$ & 4 \\
Radiation type & $\mathrm{Cu} K \alpha$ \\
$\mu\left(\mathrm{mm}^{-1}\right)$ & 0.81 \\
Crystal size (mm) & $0.50 \times 0.18 \times 0.15$ \\
& \\
Data collection & \\
Diffractometer & Enraf-Nonius CAD-4 \\
Absorption correction & $\psi$ scan $($ North et al., 1968$)$ \\
$T_{\min }, T_{\text {max }}$ & $0.688,0.888$ \\
No. of measured, independent and & $2692,2133,1808$ \\
$\quad$ observed $[I>2 \sigma(I)]$ reflections & \\
$R_{\text {int }}$ & 0.111 \\
(sin $\theta / \lambda)_{\text {max }}\left(\AA^{-1}\right)$ & 0.597 \\
Refinement & \\
$R\left[F^{2}>2 \sigma\left(F^{2}\right)\right], w R\left(F^{2}\right), S$ & $0.057,0.148,1.08$ \\
No. of reflections & 2133 \\
No. of parameters & 328 \\
No. of restraints & 1 \\
H-atom treatment & \\
$\Delta \rho_{\text {max }}, \Delta \rho_{\text {min }}\left(\mathrm{e} \AA^{-3}\right)$ & $\mathrm{H}$-atom parameters constrained \\
\hline
\end{tabular}

Computer programs: CAD-4-PC (Enraf-Nonius, 1996), REDU4 (Stoe \& Cie, 1987), SHELXS97 and SHELXL97 (Sheldrick, 2008), PLATON (Spek, 2009) and Mercury (Macrae et al., 2008)

Magotra, D. K., Gupta, V. K., Rajnikant, Goswami, K. N. \& Gupta, B. D. (1995). Acta Cryst. C51, 2637-2639.

North, A. C. T., Phillips, D. C. \& Mathews, F. S. (1968). Acta Cryst. A24, 351-359.

Schroeder, H. D., Bencze, W., Halpern, O. \& Schmid, H. (1959). Chem. Ber. 92, 2338-2363.

Scott, B. R., Pathak, M. A. \& Mohn, G. R. (1976). Mutat. Res. 39, 29 74.

Sheldrick, G. M. (2008). Acta Cryst. A64, 112-122.

Singh, A., Gupta, V. K., Kant, R. \& Goswami, K. N. (1995). Mol. Mater. 5, 289-297.

Spek, A. L. (2009). Acta Cryst. D65, 148-155.

Stemple, N. R. \& Watson, W. H. (1972). Acta Cryst. B28, 2485-2489.

Stoe \& Cie (1987). REDU4. Stoe \& Cie GmbH, Darmstadt, Germany.

Thailambal, V. G. \& Pattabhi, V. (1985). Acta Cryst. C41, 802-804.

Thailambal, V. G. \& Pattabhi, V. (1987). Acta Cryst. C43, 23692372.

Thailambal, V. G., Pattabhi, V. \& Gabe, E. J. (1986). Acta Cryst. C42, 1017-1019. 


\section{supporting information}

Acta Cryst. (2016). E72, 463-466 [https://doi.org/10.1107/S2056989016003303]

\section{Crystal structure of a photobiologically active furanocoumarin from Artemisia reticulata}

\section{A. K. Bauri, Sabine Foro and Nhu Quynh Nguyen Do}

Computing details

Data collection: $C A D-4-P C$ (Enraf-Nonius, 1996); cell refinement: CAD-4-PC (Enraf-Nonius, 1996); data reduction: REDU4 (Stoe \& Cie, 1987); program(s) used to solve structure: SHELXS97 (Sheldrick, 2008); program(s) used to refine structure: SHELXL97 (Sheldrick, 2008); molecular graphics: PLATON (Spek, 2009) and Mercury (Macrae et al., 2008); software used to prepare material for publication: SHELXL97 (Sheldrick, 2008) and PLATON (Spek, 2009).

9-Hydroxy-2-(prop-1-en-2-yl)-2,3-dihydro-7H-furo[3,2-g]chromen-7-one

Crystal data

$\mathrm{C}_{14} \mathrm{H}_{12} \mathrm{O}_{4}$

$M_{r}=244.24$

Monoclinic, $P 2_{1}$

Hall symbol: P 2y1

$a=7.2738$ (9) $\AA$

$b=21.426(2) \AA$

$c=8.0152(9) \AA$

$\beta=100.88(1)^{\circ}$

$V=1226.7(2) \AA^{3}$

$Z=4$

\section{Data collection}

Enraf-Nonius CAD-4 diffractometer

Radiation source: fine-focus sealed tube Graphite monochromator $\omega / 2 \theta$ scans

Absorption correction: $\psi$ scan

(North et al., 1968)

$T_{\min }=0.688, T_{\max }=0.888$

2692 measured reflections

\section{Refinement}

Refinement on $F^{2}$

Least-squares matrix: full

$R\left[F^{2}>2 \sigma\left(F^{2}\right)\right]=0.057$

$w R\left(F^{2}\right)=0.148$

$S=1.08$

2133 reflections

328 parameters

1 restraint
$F(000)=512$

$D_{\mathrm{x}}=1.322 \mathrm{Mg} \mathrm{m}^{-3}$

Melting point: $498 \mathrm{~K}$

$\mathrm{Cu} K \alpha$ radiation, $\lambda=1.54180 \AA$

Cell parameters from 25 reflections

$\theta=6.0-19.8^{\circ}$

$\mu=0.81 \mathrm{~mm}^{-1}$

$T=299 \mathrm{~K}$

Rod, colourless

$0.50 \times 0.18 \times 0.15 \mathrm{~mm}$

2133 independent reflections 1808 reflections with $I>2 \sigma(I)$

$R_{\text {int }}=0.111$

$\theta_{\max }=66.9^{\circ}, \theta_{\min }=4.1^{\circ}$

$h=-8 \rightarrow 8$

$k=-25 \rightarrow 0$

$l=-9 \rightarrow 2$

3 standard reflections every $120 \mathrm{~min}$ intensity decay: $1.0 \%$

Primary atom site location: structure-invariant direct methods

Secondary atom site location: difference Fourier map

Hydrogen site location: inferred from neighbouring sites

$\mathrm{H}$-atom parameters constrained 
$w=1 /\left[\sigma^{2}\left(F_{\mathrm{o}}^{2}\right)+(0.0983 P)^{2}+0.0698 P\right]$

where $P=\left(F_{\mathrm{o}}^{2}+2 F_{\mathrm{c}}{ }^{2}\right) / 3$

$(\Delta / \sigma)_{\max }=0.045$

$\Delta \rho_{\max }=0.28$ e $\AA^{-3}$
$\Delta \rho_{\min }=-0.34$ e $\AA^{-3}$

Extinction correction: SHELXL97 (Sheldrick, 2008), $\mathrm{Fc}^{*}=\mathrm{kFc}\left[1+0.001 \times \mathrm{xc}^{2} \lambda^{3} / \sin (2 \theta)\right]^{-1 / 4}$

Extinction coefficient: 0.0058 (13)

\section{Special details}

Geometry. All esds (except the esd in the dihedral angle between two 1.s. planes) are estimated using the full covariance matrix. The cell esds are taken into account individually in the estimation of esds in distances, angles and torsion angles; correlations between esds in cell parameters are only used when they are defined by crystal symmetry. An approximate (isotropic) treatment of cell esds is used for estimating esds involving l.s. planes.

Refinement. Refinement of $\mathrm{F}^{2}$ against ALL reflections. The weighted R-factor $\mathrm{wR}$ and goodness of fit $\mathrm{S}$ are based on $\mathrm{F}^{2}$, conventional $R$-factors $R$ are based on $F$, with $F$ set to zero for negative $F^{2}$. The threshold expression of $F^{2}>2 \operatorname{sigma}\left(\mathrm{F}^{2}\right)$ is used only for calculating R-factors(gt) etc. and is not relevant to the choice of reflections for refinement. R-factors based on $\mathrm{F}^{2}$ are statistically about twice as large as those based on F, and R- factors based on ALL data will be even larger.

Fractional atomic coordinates and isotropic or equivalent isotropic displacement parameters $\left(\AA^{2}\right)$

\begin{tabular}{|c|c|c|c|c|}
\hline & $x$ & $y$ & $z$ & $U_{\text {iso }} * / U_{\text {eq }}$ \\
\hline $\mathrm{O} 1$ & $0.5538(5)$ & $0.26546(17)$ & $0.2707(4)$ & $0.0718(9)$ \\
\hline $\mathrm{O} 2$ & $1.0199(4)$ & $0.35147(14)$ & $0.0003(3)$ & $0.0584(7)$ \\
\hline $\mathrm{O} 3$ & $0.8972(4)$ & $0.32756(18)$ & $0.2942(3)$ & $0.0716(9)$ \\
\hline $\mathrm{H} 3 \mathrm{O}$ & 0.9962 & 0.3467 & 0.3283 & $0.086^{*}$ \\
\hline $\mathrm{O} 4$ & $1.2459(5)$ & $0.3904(2)$ & $-0.1133(4)$ & $0.0760(9)$ \\
\hline $\mathrm{C} 1$ & $0.6289(6)$ & $0.2805(2)$ & $0.1313(5)$ & $0.0593(10)$ \\
\hline $\mathrm{C} 2$ & $0.3653(7)$ & $0.2416(2)$ & $0.2102(6)$ & $0.0677(11)$ \\
\hline $\mathrm{H} 2$ & 0.2748 & 0.2741 & 0.2241 & $0.081^{*}$ \\
\hline $\mathrm{C} 3$ & $0.3504(7)$ & $0.2283(2)$ & $0.0208(6)$ & $0.0717(12)$ \\
\hline $\mathrm{H} 3 \mathrm{~A}$ & 0.3550 & 0.1838 & -0.0007 & $0.086^{*}$ \\
\hline H3B & 0.2354 & 0.2452 & -0.0447 & $0.086^{*}$ \\
\hline $\mathrm{C} 4$ & $0.5186(6)$ & $0.2609(2)$ & $-0.0213(5)$ & $0.0615(10)$ \\
\hline $\mathrm{C} 5$ & $0.5778(7)$ & $0.2722(2)$ & $-0.1707(5)$ & $0.0646(11)$ \\
\hline H5 & 0.5045 & 0.2598 & -0.2732 & $0.078^{*}$ \\
\hline C6 & $0.7474(6)$ & $0.30226(19)$ & $-0.1693(5)$ & $0.0574(10)$ \\
\hline $\mathrm{C} 7$ & $0.8535(6)$ & $0.32065(19)$ & $-0.0126(4)$ & $0.0510(9)$ \\
\hline $\mathrm{C} 8$ & $0.7961(6)$ & $0.3100(2)$ & $0.1404(5)$ & $0.0559(10)$ \\
\hline C9 & $0.8303(7)$ & $0.3148(2)$ & $-0.3149(5)$ & $0.0643(11)$ \\
\hline H9 & 0.7672 & 0.3025 & -0.4217 & $0.077^{*}$ \\
\hline $\mathrm{C} 10$ & $0.9934(7)$ & $0.3435(2)$ & $-0.3019(5)$ & $0.0650(11)$ \\
\hline H10 & 1.0412 & 0.3510 & -0.3997 & $0.078^{*}$ \\
\hline C11 & $1.0988(7)$ & $0.3634(2)$ & $-0.1407(5)$ & $0.0608(10)$ \\
\hline $\mathrm{C} 12$ & $0.3366(8)$ & $0.1867(3)$ & $0.3189(7)$ & $0.0784(14)$ \\
\hline $\mathrm{C} 13$ & $0.4654(13)$ & $0.1340(4)$ & $0.3277(12)$ & $0.120(2)$ \\
\hline H13A & 0.4677 & 0.1193 & 0.2150 & $0.144^{*}$ \\
\hline H13B & 0.4243 & 0.1010 & 0.3929 & $0.144 *$ \\
\hline $\mathrm{H} 13 \mathrm{C}$ & 0.5888 & 0.1471 & 0.3810 & $0.144^{*}$ \\
\hline $\mathrm{C} 14$ & $0.1973(13)$ & $0.1879(5)$ & $0.4018(13)$ & $0.131(3)$ \\
\hline H14A & 0.1775 & 0.1543 & 0.4697 & $0.157^{*}$ \\
\hline H14B & 0.1184 & 0.2224 & 0.3928 & $0.157^{*}$ \\
\hline $\mathrm{O} 5$ & $0.0856(5)$ & $0.01320(19)$ & $-0.0052(4)$ & $0.0800(10)$ \\
\hline
\end{tabular}




$\begin{array}{lllll}\text { O6 } & 0.5536(4) & -0.07278(16) & 0.4256(3) & 0.0632(8) \\ \text { O7 } & 0.4356(5) & -0.04541(19) & 0.0908(3) & 0.0761(10) \\ \text { H7O } & 0.5264 & -0.0689 & 0.0873 & 0.091^{*} \\ \text { O8 } & 0.7736(5) & -0.1141(3) & 0.6163(4) & 0.0985(14) \\ \text { C15 } & 0.1590(6) & -0.0022(2) & 0.1597(5) & 0.0605(10) \\ \text { C16 } & -0.1072(8) & 0.0320(3) & -0.0122(7) & 0.0768(13) \\ \text { H16 } & -0.1887 & -0.0035 & -0.0522 & 0.092^{*} \\ \text { C17 } & -0.1231(8) & 0.0464(3) & 0.1738(7) & 0.0804(14) \\ \text { H17A } & -0.1172 & 0.0909 & 0.1960 & 0.097^{*} \\ \text { H17B } & -0.2382 & 0.0298 & 0.2005 & 0.097^{*} \\ \text { C18 } & 0.0452(6) & 0.0134(2) & 0.2722(6) & 0.0648(11) \\ \text { C19 } & 0.1022(6) & 0.0007(2) & 0.4429(6) & 0.0646(11) \\ \text { H19 } & 0.0271 & 0.0117 & 0.5200 & 0.077^{*} \\ \text { C20 } & 0.2723(6) & -0.0287(2) & 0.4982(5) & 0.0555(9) \\ \text { C21 } & 0.3829(6) & -0.04370(19) & 0.3800(5) & 0.0536(9) \\ \text { C22 } & 0.3273(6) & -0.0310(2) & 0.2071(5) & 0.0564(10) \\ \text { C23 } & 0.3455(6) & -0.0475(2) & 0.6713(5) & 0.0617(10) \\ \text { H23 } & 0.2751 & -0.0397 & 0.7547 & 0.074^{*} \\ \text { C24 } & 0.5094(7) & -0.0754(2) & 0.7141(5) & 0.0670(12) \\ \text { H24 } & 0.5520 & -0.0865 & 0.8270 & 0.080^{*} \\ \text { C25 } & 0.6234(7) & -0.0890(2) & 0.5911(5) & 0.0658(11) \\ \text { C26 } & -0.1586(9) & 0.0850(3) & -0.1320(8) & 0.0882(16) \\ \text { C27 } & -0.3538(11) & 0.0976(4) & -0.1745(13) & 0.132(3) \\ \text { H27A } & -0.4064 & 0.0762 & -0.2778 & 0.159^{*} \\ \text { H27B } & -0.3732 & 0.1417 & -0.1900 & 0.159^{*} \\ \text { H27C } & -0.4136 & 0.0834 & -0.0845 & 0.159^{*} \\ \text { C28 } & -0.0275(15) & 0.1170(6) & -0.1987(19) & 0.181(5) \\ \text { H28A } & -0.0641 & 0.1490 & -0.2764 & 0.217^{*} \\ \text { H28B } & 0.0986 & 0.1069 & -0.1666 & 0.217^{*} \\ & & & & \\ & & & & \\ \end{array}$

Atomic displacement parameters $\left(\AA^{2}\right)$

\begin{tabular}{lllllll}
\hline & $U^{11}$ & $U^{22}$ & $U^{33}$ & $U^{12}$ & $U^{13}$ & $U^{23}$ \\
\hline O1 & $0.0756(18)$ & $0.094(2)$ & $0.0482(16)$ & $-0.0229(17)$ & $0.0165(13)$ & $-0.0004(15)$ \\
O2 & $0.0673(16)$ & $0.0776(19)$ & $0.0310(12)$ & $-0.0106(14)$ & $0.0109(11)$ & $-0.0007(12)$ \\
O3 & $0.0767(18)$ & $0.107(2)$ & $0.0293(13)$ & $-0.0232(17)$ & $0.0062(12)$ & $-0.0107(14)$ \\
O4 & $0.079(2)$ & $0.104(2)$ & $0.0474(16)$ & $-0.0193(19)$ & $0.0178(14)$ & $-0.0041(16)$ \\
C1 & $0.074(2)$ & $0.066(2)$ & $0.0362(19)$ & $-0.007(2)$ & $0.0076(17)$ & $-0.0017(18)$ \\
C2 & $0.072(3)$ & $0.073(3)$ & $0.059(3)$ & $-0.017(2)$ & $0.014(2)$ & $-0.003(2)$ \\
C3 & $0.077(3)$ & $0.074(3)$ & $0.059(2)$ & $-0.016(2)$ & $0.000(2)$ & $0.001(2)$ \\
C4 & $0.070(2)$ & $0.058(2)$ & $0.052(2)$ & $-0.0097(19)$ & $-0.0007(18)$ & $-0.0027(17)$ \\
C5 & $0.087(3)$ & $0.066(3)$ & $0.0349(19)$ & $-0.006(2)$ & $-0.0039(18)$ & $-0.0053(18)$ \\
C6 & $0.075(3)$ & $0.059(2)$ & $0.0347(19)$ & $-0.0034(19)$ & $0.0009(16)$ & $-0.0010(16)$ \\
C7 & $0.067(2)$ & $0.057(2)$ & $0.0281(17)$ & $-0.0028(18)$ & $0.0071(14)$ & $-0.0035(14)$ \\
C8 & $0.065(2)$ & $0.067(2)$ & $0.0328(17)$ & $-0.0066(19)$ & $0.0005(16)$ & $-0.0047(17)$ \\
C9 & $0.092(3)$ & $0.069(3)$ & $0.0301(17)$ & $-0.002(2)$ & $0.0067(17)$ & $0.0000(18)$ \\
C10 & $0.087(3)$ & $0.074(3)$ & $0.0349(19)$ & $-0.007(2)$ & $0.0131(19)$ & $0.0024(18)$ \\
C11 & $0.075(3)$ & $0.073(3)$ & $0.0370(19)$ & $0.001(2)$ & $0.0156(18)$ & $-0.0007(17)$
\end{tabular}




$\begin{array}{lllllll}\text { C12 } & 0.089(3) & 0.081(3) & 0.067(3) & -0.022(3) & 0.021(3) & -0.003(2) \\ \text { C13 } & 0.146(6) & 0.094(5) & 0.120(6) & 0.009(5) & 0.023(5) & 0.028(4) \\ \text { C14 } & 0.140(6) & 0.125(6) & 0.144(8) & -0.029(5) & 0.066(6) & 0.018(5) \\ \text { O5 } & 0.087(2) & 0.104(3) & 0.0412(15) & 0.024(2) & -0.0062(14) & 0.0061(15) \\ \text { O6 } & 0.0667(16) & 0.092(2) & 0.0293(13) & 0.0173(15) & 0.0059(11) & 0.0098(13) \\ \text { O7 } & 0.085(2) & 0.111(3) & 0.0339(14) & 0.0274(19) & 0.0166(13) & 0.0096(15) \\ \text { O8 } & 0.091(2) & 0.156(4) & 0.0462(18) & 0.048(3) & 0.0075(16) & 0.026(2) \\ \text { C15 } & 0.069(2) & 0.069(2) & 0.039(2) & 0.004(2) & -0.0011(17) & 0.0035(18) \\ \text { C16 } & 0.080(3) & 0.079(3) & 0.063(3) & 0.008(3) & -0.009(2) & 0.001(2) \\ \text { C17 } & 0.073(3) & 0.092(3) & 0.074(3) & 0.019(3) & 0.006(2) & -0.003(3) \\ \text { C18 } & 0.065(2) & 0.069(3) & 0.057(3) & 0.006(2) & 0.0030(19) & -0.003(2) \\ \text { C19 } & 0.069(2) & 0.074(3) & 0.052(2) & 0.005(2) & 0.0156(19) & -0.006(2) \\ \text { C20 } & 0.066(2) & 0.063(2) & 0.0377(19) & -0.0015(19) & 0.0103(16) & -0.0041(16) \\ \text { C21 } & 0.065(2) & 0.062(2) & 0.0330(18) & 0.0021(18) & 0.0053(15) & 0.0023(15) \\ \text { C22 } & 0.067(2) & 0.067(2) & 0.0334(18) & 0.0069(19) & 0.0049(16) & 0.0051(16) \\ \text { C23 } & 0.081(3) & 0.075(3) & 0.0317(18) & 0.000(2) & 0.0183(17) & -0.0042(17) \\ \text { C24 } & 0.082(3) & 0.085(3) & 0.0320(19) & 0.002(2) & 0.0072(18) & 0.0045(19) \\ \text { C25 } & 0.076(3) & 0.088(3) & 0.0319(18) & 0.012(2) & 0.0070(18) & 0.0117(18) \\ \text { C26 } & 0.096(4) & 0.068(3) & 0.087(3) & 0.010(3) & -0.016(3) & 0.000(3) \\ \text { C27 } & 0.111(5) & 0.114(6) & 0.152(7) & 0.017(4) & -0.025(5) & 0.034(5) \\ \text { C28 } & 0.136(7) & 0.150(8) & 0.241(13) & -0.008(7) & -0.003(8) & 0.106(9)\end{array}$

Geometric parameters $\left(\AA,{ }^{\circ}\right)$

\begin{tabular}{|c|c|c|c|}
\hline $\mathrm{O} 1-\mathrm{C} 1$ & $1.371(5)$ & $\mathrm{O} 5-\mathrm{C} 15$ & $1.369(5)$ \\
\hline $\mathrm{O} 1-\mathrm{C} 2$ & $1.459(5)$ & $\mathrm{O} 5-\mathrm{C} 16$ & $1.449(6)$ \\
\hline $\mathrm{O} 2-\mathrm{C} 7$ & $1.366(5)$ & $\mathrm{O} 6-\mathrm{C} 25$ & $1.373(5)$ \\
\hline $\mathrm{O} 2-\mathrm{C} 11$ & $1.384(5)$ & $\mathrm{O} 6-\mathrm{C} 21$ & $1.375(5)$ \\
\hline $\mathrm{O} 3-\mathrm{C} 8$ & $1.363(5)$ & $\mathrm{O} 7-\mathrm{C} 22$ & $1.365(5)$ \\
\hline $\mathrm{O} 3-\mathrm{H} 3 \mathrm{O}$ & 0.8294 & $\mathrm{O} 7-\mathrm{H} 7 \mathrm{O}$ & 0.8354 \\
\hline $\mathrm{O} 4-\mathrm{C} 11$ & $1.199(5)$ & $\mathrm{O} 8-\mathrm{C} 25$ & $1.200(6)$ \\
\hline $\mathrm{C} 1-\mathrm{C} 8$ & $1.361(6)$ & $\mathrm{C} 15-\mathrm{C} 22$ & $1.360(6)$ \\
\hline $\mathrm{C} 1-\mathrm{C} 4$ & $1.395(6)$ & $\mathrm{C} 15-\mathrm{C} 18$ & $1.376(7)$ \\
\hline $\mathrm{C} 2-\mathrm{C} 12$ & $1.500(8)$ & $\mathrm{C} 16-\mathrm{C} 26$ & $1.489(8)$ \\
\hline $\mathrm{C} 2-\mathrm{C} 3$ & $1.529(7)$ & $\mathrm{C} 16-\mathrm{C} 17$ & $1.549(8)$ \\
\hline $\mathrm{C} 2-\mathrm{H} 2$ & 0.9800 & $\mathrm{C} 16-\mathrm{H} 16$ & 0.9800 \\
\hline $\mathrm{C} 3-\mathrm{C} 4$ & $1.501(7)$ & $\mathrm{C} 17-\mathrm{C} 18$ & $1.502(7)$ \\
\hline $\mathrm{C} 3-\mathrm{H} 3 \mathrm{~A}$ & 0.9700 & C17-H17A & 0.9700 \\
\hline $\mathrm{C} 3-\mathrm{H} 3 \mathrm{~B}$ & 0.9700 & $\mathrm{C} 17-\mathrm{H} 17 \mathrm{~B}$ & 0.9700 \\
\hline $\mathrm{C} 4-\mathrm{C} 5$ & $1.369(7)$ & $\mathrm{C} 18-\mathrm{C} 19$ & $1.379(6)$ \\
\hline $\mathrm{C} 5-\mathrm{C} 6$ & $1.389(7)$ & $\mathrm{C} 19-\mathrm{C} 20$ & $1.384(6)$ \\
\hline $\mathrm{C} 5-\mathrm{H} 5$ & 0.9300 & C19-H19 & 0.9300 \\
\hline $\mathrm{C} 6-\mathrm{C} 7$ & $1.400(5)$ & $\mathrm{C} 20-\mathrm{C} 21$ & $1.391(6)$ \\
\hline $\mathrm{C} 6-\mathrm{C} 9$ & $1.437(7)$ & $\mathrm{C} 20-\mathrm{C} 23$ & $1.447(5)$ \\
\hline $\mathrm{C} 7-\mathrm{C} 8$ & $1.387(6)$ & $\mathrm{C} 21-\mathrm{C} 22$ & $1.395(5)$ \\
\hline $\mathrm{C} 9-\mathrm{C} 10$ & $1.322(7)$ & $\mathrm{C} 23-\mathrm{C} 24$ & $1.320(7)$ \\
\hline $\mathrm{C} 9-\mathrm{H} 9$ & 0.9300 & $\mathrm{C} 23-\mathrm{H} 23$ & 0.9300 \\
\hline $\mathrm{C} 10-\mathrm{C} 11$ & $1.437(6)$ & $\mathrm{C} 24-\mathrm{C} 25$ & $1.433(7)$ \\
\hline
\end{tabular}




\begin{tabular}{|c|c|c|c|}
\hline $\mathrm{C} 10-\mathrm{H} 10$ & 0.9300 & $\mathrm{C} 24-\mathrm{H} 24$ & 0.9300 \\
\hline $\mathrm{C} 12-\mathrm{C} 14$ & $1.313(10)$ & $\mathrm{C} 26-\mathrm{C} 28$ & $1.363(13)$ \\
\hline $\mathrm{C} 12-\mathrm{C} 13$ & $1.461(10)$ & $\mathrm{C} 26-\mathrm{C} 27$ & $1.422(10)$ \\
\hline C13-H13A & 0.9600 & $\mathrm{C} 27-\mathrm{H} 27 \mathrm{~A}$ & 0.9600 \\
\hline C13-H13B & 0.9600 & $\mathrm{C} 27-\mathrm{H} 27 \mathrm{~B}$ & 0.9600 \\
\hline $\mathrm{C} 13-\mathrm{H} 13 \mathrm{C}$ & 0.9600 & $\mathrm{C} 27-\mathrm{H} 27 \mathrm{C}$ & 0.9600 \\
\hline $\mathrm{C} 14-\mathrm{H} 14 \mathrm{~A}$ & 0.9300 & $\mathrm{C} 28-\mathrm{H} 28 \mathrm{~A}$ & 0.9300 \\
\hline C14-H14B & 0.9300 & $\mathrm{C} 28-\mathrm{H} 28 \mathrm{~B}$ & 0.9300 \\
\hline $\mathrm{C} 1-\mathrm{O} 1-\mathrm{C} 2$ & $107.8(3)$ & $\mathrm{C} 15-\mathrm{O} 5-\mathrm{C} 16$ & $107.6(4)$ \\
\hline $\mathrm{C} 7-\mathrm{O} 2-\mathrm{C} 11$ & $121.7(3)$ & $\mathrm{C} 25-\mathrm{O} 6-\mathrm{C} 21$ & $121.5(3)$ \\
\hline $\mathrm{C} 8-\mathrm{O} 3-\mathrm{H} 3 \mathrm{O}$ & 136.1 & $\mathrm{C} 22-\mathrm{O} 7-\mathrm{H} 7 \mathrm{O}$ & 136.1 \\
\hline $\mathrm{C} 8-\mathrm{C} 1-\mathrm{O} 1$ & $123.7(3)$ & $\mathrm{C} 22-\mathrm{C} 15-\mathrm{O} 5$ & $123.1(4)$ \\
\hline $\mathrm{C} 8-\mathrm{C} 1-\mathrm{C} 4$ & $123.2(4)$ & $\mathrm{C} 22-\mathrm{C} 15-\mathrm{C} 18$ & $123.2(4)$ \\
\hline $\mathrm{O} 1-\mathrm{C} 1-\mathrm{C} 4$ & $113.1(4)$ & $\mathrm{O} 5-\mathrm{C} 15-\mathrm{C} 18$ & $113.7(4)$ \\
\hline $\mathrm{O} 1-\mathrm{C} 2-\mathrm{C} 12$ & $107.9(4)$ & $\mathrm{O} 5-\mathrm{C} 16-\mathrm{C} 26$ & $111.1(5)$ \\
\hline $\mathrm{O} 1-\mathrm{C} 2-\mathrm{C} 3$ & $106.3(4)$ & $\mathrm{O} 5-\mathrm{C} 16-\mathrm{C} 17$ & $105.4(3)$ \\
\hline $\mathrm{C} 12-\mathrm{C} 2-\mathrm{C} 3$ & $116.1(4)$ & $\mathrm{C} 26-\mathrm{C} 16-\mathrm{C} 17$ & $114.4(5)$ \\
\hline $\mathrm{O} 1-\mathrm{C} 2-\mathrm{H} 2$ & 108.8 & $\mathrm{O} 5-\mathrm{C} 16-\mathrm{H} 16$ & 108.6 \\
\hline $\mathrm{C} 12-\mathrm{C} 2-\mathrm{H} 2$ & 108.8 & $\mathrm{C} 26-\mathrm{C} 16-\mathrm{H} 16$ & 108.6 \\
\hline $\mathrm{C} 3-\mathrm{C} 2-\mathrm{H} 2$ & 108.8 & $\mathrm{C} 17-\mathrm{C} 16-\mathrm{H} 16$ & 108.6 \\
\hline $\mathrm{C} 4-\mathrm{C} 3-\mathrm{C} 2$ & $103.2(3)$ & $\mathrm{C} 18-\mathrm{C} 17-\mathrm{C} 16$ & $102.1(4)$ \\
\hline $\mathrm{C} 4-\mathrm{C} 3-\mathrm{H} 3 \mathrm{~A}$ & 111.1 & $\mathrm{C} 18-\mathrm{C} 17-\mathrm{H} 17 \mathrm{~A}$ & 111.3 \\
\hline $\mathrm{C} 2-\mathrm{C} 3-\mathrm{H} 3 \mathrm{~A}$ & 111.1 & $\mathrm{C} 16-\mathrm{C} 17-\mathrm{H} 17 \mathrm{~A}$ & 111.3 \\
\hline $\mathrm{C} 4-\mathrm{C} 3-\mathrm{H} 3 \mathrm{~B}$ & 111.1 & $\mathrm{C} 18-\mathrm{C} 17-\mathrm{H} 17 \mathrm{~B}$ & 111.3 \\
\hline $\mathrm{C} 2-\mathrm{C} 3-\mathrm{H} 3 \mathrm{~B}$ & 111.1 & $\mathrm{C} 16-\mathrm{C} 17-\mathrm{H} 17 \mathrm{~B}$ & 111.3 \\
\hline $\mathrm{H} 3 \mathrm{~A}-\mathrm{C} 3-\mathrm{H} 3 \mathrm{~B}$ & 109.1 & $\mathrm{H} 17 \mathrm{~A}-\mathrm{C} 17-\mathrm{H} 17 \mathrm{~B}$ & 109.2 \\
\hline $\mathrm{C} 5-\mathrm{C} 4-\mathrm{C} 1$ & $119.3(4)$ & $\mathrm{C} 15-\mathrm{C} 18-\mathrm{C} 19$ & $119.8(4)$ \\
\hline $\mathrm{C} 5-\mathrm{C} 4-\mathrm{C} 3$ & $133.1(4)$ & $\mathrm{C} 15-\mathrm{C} 18-\mathrm{C} 17$ & $107.5(4)$ \\
\hline $\mathrm{C} 1-\mathrm{C} 4-\mathrm{C} 3$ & $107.6(4)$ & $\mathrm{C} 19-\mathrm{C} 18-\mathrm{C} 17$ & $132.6(4)$ \\
\hline $\mathrm{C} 4-\mathrm{C} 5-\mathrm{C} 6$ & $120.0(4)$ & $\mathrm{C} 18-\mathrm{C} 19-\mathrm{C} 20$ & $119.4(4)$ \\
\hline $\mathrm{C} 4-\mathrm{C} 5-\mathrm{H} 5$ & 120.0 & $\mathrm{C} 18-\mathrm{C} 19-\mathrm{H} 19$ & 120.3 \\
\hline $\mathrm{C} 6-\mathrm{C} 5-\mathrm{H} 5$ & 120.0 & $\mathrm{C} 20-\mathrm{C} 19-\mathrm{H} 19$ & 120.3 \\
\hline $\mathrm{C} 5-\mathrm{C} 6-\mathrm{C} 7$ & $118.4(4)$ & $\mathrm{C} 19-\mathrm{C} 20-\mathrm{C} 21$ & $118.9(4)$ \\
\hline $\mathrm{C} 5-\mathrm{C} 6-\mathrm{C} 9$ & $126.0(4)$ & $\mathrm{C} 19-\mathrm{C} 20-\mathrm{C} 23$ & $125.3(4)$ \\
\hline $\mathrm{C} 7-\mathrm{C} 6-\mathrm{C} 9$ & $115.5(4)$ & $\mathrm{C} 21-\mathrm{C} 20-\mathrm{C} 23$ & $115.7(4)$ \\
\hline $\mathrm{O} 2-\mathrm{C} 7-\mathrm{C} 8$ & $115.0(3)$ & $\mathrm{O} 6-\mathrm{C} 21-\mathrm{C} 20$ & $122.2(3)$ \\
\hline $\mathrm{O} 2-\mathrm{C} 7-\mathrm{C} 6$ & $122.3(3)$ & $\mathrm{O} 6-\mathrm{C} 21-\mathrm{C} 22$ & $115.5(3)$ \\
\hline $\mathrm{C} 8-\mathrm{C} 7-\mathrm{C} 6$ & $122.7(4)$ & $\mathrm{C} 20-\mathrm{C} 21-\mathrm{C} 22$ & $122.3(4)$ \\
\hline $\mathrm{O} 3-\mathrm{C} 8-\mathrm{C} 1$ & $120.0(4)$ & $\mathrm{C} 15-\mathrm{C} 22-\mathrm{O} 7$ & $121.1(3)$ \\
\hline $\mathrm{O} 3-\mathrm{C} 8-\mathrm{C} 7$ & $123.6(4)$ & $\mathrm{C} 15-\mathrm{C} 22-\mathrm{C} 21$ & $116.3(4)$ \\
\hline $\mathrm{C} 1-\mathrm{C} 8-\mathrm{C} 7$ & $116.4(3)$ & $\mathrm{O} 7-\mathrm{C} 22-\mathrm{C} 21$ & $122.6(4)$ \\
\hline $\mathrm{C} 10-\mathrm{C} 9-\mathrm{C} 6$ & $122.1(4)$ & $\mathrm{C} 24-\mathrm{C} 23-\mathrm{C} 20$ & $121.7(4)$ \\
\hline $\mathrm{C} 10-\mathrm{C} 9-\mathrm{H} 9$ & 119.0 & $\mathrm{C} 24-\mathrm{C} 23-\mathrm{H} 23$ & 119.1 \\
\hline $\mathrm{C} 6-\mathrm{C} 9-\mathrm{H} 9$ & 119.0 & $\mathrm{C} 20-\mathrm{C} 23-\mathrm{H} 23$ & 119.1 \\
\hline $\mathrm{C} 9-\mathrm{C} 10-\mathrm{C} 11$ & $121.8(4)$ & $\mathrm{C} 23-\mathrm{C} 24-\mathrm{C} 25$ & $121.7(4)$ \\
\hline $\mathrm{C} 9-\mathrm{C} 10-\mathrm{H} 10$ & 119.1 & $\mathrm{C} 23-\mathrm{C} 24-\mathrm{H} 24$ & 119.2 \\
\hline $\mathrm{C} 11-\mathrm{C} 10-\mathrm{H} 10$ & 119.1 & $\mathrm{C} 25-\mathrm{C} 24-\mathrm{H} 24$ & 119.2 \\
\hline
\end{tabular}




\begin{tabular}{|c|c|c|c|}
\hline $\mathrm{O} 4-\mathrm{C} 11-\mathrm{O} 2$ & $115.6(4)$ & $\mathrm{O} 8-\mathrm{C} 25-\mathrm{O} 6$ & $115.8(4)$ \\
\hline $\mathrm{O} 4-\mathrm{C} 11-\mathrm{C} 10$ & $127.9(4)$ & $\mathrm{O} 8-\mathrm{C} 25-\mathrm{C} 24$ & $127.0(4)$ \\
\hline $\mathrm{O} 2-\mathrm{C} 11-\mathrm{C} 10$ & $116.5(4)$ & $\mathrm{O} 6-\mathrm{C} 25-\mathrm{C} 24$ & $117.2(4)$ \\
\hline $\mathrm{C} 14-\mathrm{C} 12-\mathrm{C} 13$ & $122.7(7)$ & $\mathrm{C} 28-\mathrm{C} 26-\mathrm{C} 27$ & $123.5(7)$ \\
\hline $\mathrm{C} 14-\mathrm{C} 12-\mathrm{C} 2$ & $118.9(7)$ & $\mathrm{C} 28-\mathrm{C} 26-\mathrm{C} 16$ & $121.9(6)$ \\
\hline $\mathrm{C} 13-\mathrm{C} 12-\mathrm{C} 2$ & $118.4(5)$ & $\mathrm{C} 27-\mathrm{C} 26-\mathrm{C} 16$ & $114.7(6)$ \\
\hline $\mathrm{C} 12-\mathrm{C} 13-\mathrm{H} 13 \mathrm{~A}$ & 109.5 & $\mathrm{C} 26-\mathrm{C} 27-\mathrm{H} 27 \mathrm{~A}$ & 109.5 \\
\hline $\mathrm{C} 12-\mathrm{C} 13-\mathrm{H} 13 \mathrm{~B}$ & 109.5 & $\mathrm{C} 26-\mathrm{C} 27-\mathrm{H} 27 \mathrm{~B}$ & 109.5 \\
\hline $\mathrm{H} 13 \mathrm{~A}-\mathrm{C} 13-\mathrm{H} 13 \mathrm{~B}$ & 109.5 & $\mathrm{H} 27 \mathrm{~A}-\mathrm{C} 27-\mathrm{H} 27 \mathrm{~B}$ & 109.5 \\
\hline $\mathrm{C} 12-\mathrm{C} 13-\mathrm{H} 13 \mathrm{C}$ & 109.5 & $\mathrm{C} 26-\mathrm{C} 27-\mathrm{H} 27 \mathrm{C}$ & 109.5 \\
\hline $\mathrm{H} 13 \mathrm{~A}-\mathrm{C} 13-\mathrm{H} 13 \mathrm{C}$ & 109.5 & $\mathrm{H} 27 \mathrm{~A}-\mathrm{C} 27-\mathrm{H} 27 \mathrm{C}$ & 109.5 \\
\hline $\mathrm{H} 13 \mathrm{~B}-\mathrm{C} 13-\mathrm{H} 13 \mathrm{C}$ & 109.5 & $\mathrm{H} 27 \mathrm{~B}-\mathrm{C} 27-\mathrm{H} 27 \mathrm{C}$ & 109.5 \\
\hline $\mathrm{C} 12-\mathrm{C} 14-\mathrm{H} 14 \mathrm{~A}$ & 120.0 & $\mathrm{C} 26-\mathrm{C} 28-\mathrm{H} 28 \mathrm{~A}$ & 120.0 \\
\hline $\mathrm{C} 12-\mathrm{C} 14-\mathrm{H} 14 \mathrm{~B}$ & 120.0 & $\mathrm{C} 26-\mathrm{C} 28-\mathrm{H} 28 \mathrm{~B}$ & 120.0 \\
\hline $\mathrm{H} 14 \mathrm{~A}-\mathrm{C} 14-\mathrm{H} 14 \mathrm{~B}$ & 120.0 & $\mathrm{H} 28 \mathrm{~A}-\mathrm{C} 28-\mathrm{H} 28 \mathrm{~B}$ & 120.0 \\
\hline $\mathrm{C} 2-\mathrm{O} 1-\mathrm{C} 1-\mathrm{C} 8$ & $173.1(5)$ & $\mathrm{C} 16-\mathrm{O} 5-\mathrm{C} 15-\mathrm{C} 22$ & $169.4(5)$ \\
\hline $\mathrm{C} 2-\mathrm{O} 1-\mathrm{C} 1-\mathrm{C} 4$ & $-8.5(6)$ & $\mathrm{C} 16-\mathrm{O} 5-\mathrm{C} 15-\mathrm{C} 18$ & $-9.7(6)$ \\
\hline $\mathrm{C} 1-\mathrm{O} 1-\mathrm{C} 2-\mathrm{C} 12$ & $139.0(4)$ & $\mathrm{C} 15-\mathrm{O} 5-\mathrm{C} 16-\mathrm{C} 26$ & $142.2(4)$ \\
\hline $\mathrm{C} 1-\mathrm{O} 1-\mathrm{C} 2-\mathrm{C} 3$ & $13.8(5)$ & $\mathrm{C} 15-\mathrm{O} 5-\mathrm{C} 16-\mathrm{C} 17$ & $17.7(6)$ \\
\hline $\mathrm{O} 1-\mathrm{C} 2-\mathrm{C} 3-\mathrm{C} 4$ & $-13.6(5)$ & $\mathrm{O} 5-\mathrm{C} 16-\mathrm{C} 17-\mathrm{C} 18$ & $-18.6(6)$ \\
\hline $\mathrm{C} 12-\mathrm{C} 2-\mathrm{C} 3-\mathrm{C} 4$ & $-133.6(5)$ & $\mathrm{C} 26-\mathrm{C} 16-\mathrm{C} 17-\mathrm{C} 18$ & $-141.0(5)$ \\
\hline $\mathrm{C} 8-\mathrm{C} 1-\mathrm{C} 4-\mathrm{C} 5$ & $-0.9(7)$ & $\mathrm{C} 22-\mathrm{C} 15-\mathrm{C} 18-\mathrm{C} 19$ & $1.1(7)$ \\
\hline $\mathrm{O} 1-\mathrm{C} 1-\mathrm{C} 4-\mathrm{C} 5$ & $-179.3(4)$ & $\mathrm{O} 5-\mathrm{C} 15-\mathrm{C} 18-\mathrm{C} 19$ & $-179.8(4)$ \\
\hline $\mathrm{C} 8-\mathrm{C} 1-\mathrm{C} 4-\mathrm{C} 3$ & $177.8(5)$ & $\mathrm{C} 22-\mathrm{C} 15-\mathrm{C} 18-\mathrm{C} 17$ & $177.9(5)$ \\
\hline $\mathrm{O} 1-\mathrm{C} 1-\mathrm{C} 4-\mathrm{C} 3$ & $-0.6(6)$ & $\mathrm{O} 5-\mathrm{C} 15-\mathrm{C} 18-\mathrm{C} 17$ & $-3.1(6)$ \\
\hline $\mathrm{C} 2-\mathrm{C} 3-\mathrm{C} 4-\mathrm{C} 5$ & $-172.7(5)$ & $\mathrm{C} 16-\mathrm{C} 17-\mathrm{C} 18-\mathrm{C} 15$ & $13.4(6)$ \\
\hline $\mathrm{C} 2-\mathrm{C} 3-\mathrm{C} 4-\mathrm{C} 1$ & $8.8(5)$ & $\mathrm{C} 16-\mathrm{C} 17-\mathrm{C} 18-\mathrm{C} 19$ & $-170.5(5)$ \\
\hline $\mathrm{C} 1-\mathrm{C} 4-\mathrm{C} 5-\mathrm{C} 6$ & $0.9(7)$ & $\mathrm{C} 15-\mathrm{C} 18-\mathrm{C} 19-\mathrm{C} 20$ & $-1.1(7)$ \\
\hline $\mathrm{C} 3-\mathrm{C} 4-\mathrm{C} 5-\mathrm{C} 6$ & $-177.5(5)$ & $\mathrm{C} 17-\mathrm{C} 18-\mathrm{C} 19-\mathrm{C} 20$ & $-176.9(5)$ \\
\hline $\mathrm{C} 4-\mathrm{C} 5-\mathrm{C} 6-\mathrm{C} 7$ & $-0.4(6)$ & $\mathrm{C} 18-\mathrm{C} 19-\mathrm{C} 20-\mathrm{C} 21$ & $1.0(7)$ \\
\hline $\mathrm{C} 4-\mathrm{C} 5-\mathrm{C} 6-\mathrm{C} 9$ & $177.3(4)$ & $\mathrm{C} 18-\mathrm{C} 19-\mathrm{C} 20-\mathrm{C} 23$ & $-176.9(4)$ \\
\hline $\mathrm{C} 11-\mathrm{O} 2-\mathrm{C} 7-\mathrm{C} 8$ & $177.6(4)$ & $\mathrm{C} 25-\mathrm{O} 6-\mathrm{C} 21-\mathrm{C} 20$ & $0.5(6)$ \\
\hline $\mathrm{C} 11-\mathrm{O} 2-\mathrm{C} 7-\mathrm{C} 6$ & $-3.6(6)$ & $\mathrm{C} 25-\mathrm{O} 6-\mathrm{C} 21-\mathrm{C} 22$ & $-178.7(4)$ \\
\hline $\mathrm{C} 5-\mathrm{C} 6-\mathrm{C} 7-\mathrm{O} 2$ & $-178.7(4)$ & $\mathrm{C} 19-\mathrm{C} 20-\mathrm{C} 21-\mathrm{O} 6$ & $179.9(4)$ \\
\hline $\mathrm{C} 9-\mathrm{C} 6-\mathrm{C} 7-\mathrm{O} 2$ & $3.4(6)$ & $\mathrm{C} 23-\mathrm{C} 20-\mathrm{C} 21-\mathrm{O} 6$ & $-2.0(6)$ \\
\hline $\mathrm{C} 5-\mathrm{C} 6-\mathrm{C} 7-\mathrm{C} 8$ & $0.0(6)$ & $\mathrm{C} 19-\mathrm{C} 20-\mathrm{C} 21-\mathrm{C} 22$ & $-1.0(7)$ \\
\hline $\mathrm{C} 9-\mathrm{C} 6-\mathrm{C} 7-\mathrm{C} 8$ & $-177.9(4)$ & $\mathrm{C} 23-\mathrm{C} 20-\mathrm{C} 21-\mathrm{C} 22$ & $177.2(4)$ \\
\hline $\mathrm{O} 1-\mathrm{C} 1-\mathrm{C} 8-\mathrm{O} 3$ & $-1.3(7)$ & $\mathrm{O} 5-\mathrm{C} 15-\mathrm{C} 22-\mathrm{O} 7$ & $1.2(7)$ \\
\hline $\mathrm{C} 4-\mathrm{C} 1-\mathrm{C} 8-\mathrm{O} 3$ & $-179.6(4)$ & $\mathrm{C} 18-\mathrm{C} 15-\mathrm{C} 22-\mathrm{O} 7$ & $-179.8(4)$ \\
\hline $\mathrm{O} 1-\mathrm{C} 1-\mathrm{C} 8-\mathrm{C} 7$ & $178.7(4)$ & $\mathrm{O} 5-\mathrm{C} 15-\mathrm{C} 22-\mathrm{C} 21$ & $-180.0(4)$ \\
\hline $\mathrm{C} 4-\mathrm{C} 1-\mathrm{C} 8-\mathrm{C} 7$ & $0.5(7)$ & $\mathrm{C} 18-\mathrm{C} 15-\mathrm{C} 22-\mathrm{C} 21$ & $-1.0(7)$ \\
\hline $\mathrm{O} 2-\mathrm{C} 7-\mathrm{C} 8-\mathrm{O} 3$ & $-1.2(6)$ & $\mathrm{O} 6-\mathrm{C} 21-\mathrm{C} 22-\mathrm{C} 15$ & $-179.9(4)$ \\
\hline $\mathrm{C} 6-\mathrm{C} 7-\mathrm{C} 8-\mathrm{O} 3$ & $-180.0(4)$ & $\mathrm{C} 20-\mathrm{C} 21-\mathrm{C} 22-\mathrm{C} 15$ & $0.9(6)$ \\
\hline $\mathrm{O} 2-\mathrm{C} 7-\mathrm{C} 8-\mathrm{C} 1$ & $178.8(4)$ & $\mathrm{O} 6-\mathrm{C} 21-\mathrm{C} 22-\mathrm{O} 7$ & $-1.1(6)$ \\
\hline $\mathrm{C} 6-\mathrm{C} 7-\mathrm{C} 8-\mathrm{C} 1$ & $-0.1(6)$ & $\mathrm{C} 20-\mathrm{C} 21-\mathrm{C} 22-\mathrm{O} 7$ & $179.7(4)$ \\
\hline $\mathrm{C} 5-\mathrm{C} 6-\mathrm{C} 9-\mathrm{C} 10$ & $-179.6(5)$ & $\mathrm{C} 19-\mathrm{C} 20-\mathrm{C} 23-\mathrm{C} 24$ & $180.0(5)$ \\
\hline $\mathrm{C} 7-\mathrm{C} 6-\mathrm{C} 9-\mathrm{C} 10$ & $-1.9(7)$ & $\mathrm{C} 21-\mathrm{C} 20-\mathrm{C} 23-\mathrm{C} 24$ & $2.0(6)$ \\
\hline
\end{tabular}




$\begin{array}{llll}\mathrm{C} 6-\mathrm{C} 9-\mathrm{C} 10-\mathrm{C} 11 & 0.5(7) & \mathrm{C} 20-\mathrm{C} 23-\mathrm{C} 24-\mathrm{C} 25 & -0.5(8) \\ \mathrm{C} 7-\mathrm{O} 2-\mathrm{C} 11-\mathrm{O} 4 & -179.5(4) & \mathrm{C} 21-\mathrm{O} 6-\mathrm{C} 25-\mathrm{O} 8 & -180.0(5) \\ \mathrm{C} 7-\mathrm{O} 2-\mathrm{C} 11-\mathrm{C} 10 & 2.0(6) & \mathrm{C} 21-\mathrm{O} 6-\mathrm{C} 25-\mathrm{C} 24 & 1.1(7) \\ \mathrm{C} 9-\mathrm{C} 10-\mathrm{C} 11-\mathrm{O} 4 & -178.8(5) & \mathrm{C} 23-\mathrm{C} 24-\mathrm{C} 25-\mathrm{O} 8 & -179.9(6) \\ \mathrm{C} 9-\mathrm{C} 10-\mathrm{C} 11-\mathrm{O} 2 & -0.5(7) & \mathrm{C} 23-\mathrm{C} 24-\mathrm{C} 25-\mathrm{O} 6 & -1.1(8) \\ \mathrm{O} 1-\mathrm{C} 2-\mathrm{C} 12-\mathrm{C} 14 & 122.2(7) & \mathrm{O} 5-\mathrm{C} 16-\mathrm{C} 26-\mathrm{C} 28 & -10.8(11) \\ \mathrm{C} 3-\mathrm{C} 2-\mathrm{C} 12-\mathrm{C} 14 & -118.7(7) & \mathrm{C} 17-\mathrm{C} 16-\mathrm{C} 26-\mathrm{C} 28 & 108.4(10) \\ \mathrm{O} 1-\mathrm{C} 2-\mathrm{C} 12-\mathrm{C} 13 & -57.9(7) & \mathrm{O} 5-\mathrm{C} 16-\mathrm{C} 26-\mathrm{C} 27 & 167.1(6) \\ \mathrm{C} 3-\mathrm{C} 2-\mathrm{C} 12-\mathrm{C} 13 & 61.3(7) & \mathrm{C} 17-\mathrm{C} 16-\mathrm{C} 26-\mathrm{C} 27 & -73.7(8)\end{array}$

Hydrogen-bond geometry $\left(A,{ }^{\circ}\right)$

$\mathrm{Cg} 2$ and $\mathrm{Cg} 9$ are the centroids of rings $\mathrm{O} 2 / \mathrm{C} 6 / \mathrm{C} 7 / \mathrm{C} 9-\mathrm{C} 11$ and $\mathrm{C} 15-\mathrm{C} 22$, respectively.

\begin{tabular}{lllll}
\hline$D-\mathrm{H} \cdots A$ & $D-\mathrm{H}$ & $\mathrm{H} \cdots A$ & $D \cdots A$ & $D-\mathrm{H} \cdots A$ \\
\hline $\mathrm{O} 3-\mathrm{H} 3 O \cdots \mathrm{O}^{\mathrm{i}}$ & 0.83 & 1.85 & $2.676(5)$ & 174 \\
$\mathrm{O} 7-\mathrm{H} 7 O \cdots{ }^{\text {ii }}$ & 0.84 & 1.85 & $2.671(5)$ & 168 \\
$\mathrm{C} 10-\mathrm{H} 10 \cdots 3^{\mathrm{iii}}$ & 0.93 & 2.53 & $3.199(5)$ & 129 \\
$\mathrm{C} 10-\mathrm{H} 10 \cdots 8^{\text {iv }}$ & 0.93 & 2.50 & $3.415(6)$ & 166 \\
$\mathrm{C} 24-\mathrm{H} 24 \cdots \mathrm{O} 7^{\mathrm{v}}$ & 0.93 & 2.58 & $3.229(5)$ & 128 \\
$\mathrm{C} 24-\mathrm{H} 24 \cdots \mathrm{O} 4^{\mathrm{vi}}$ & 0.93 & 2.53 & $3.434(5)$ & 164 \\
$\mathrm{C} 3-\mathrm{H} 3 B \cdots C g 2^{\mathrm{vii}}$ & 0.97 & 2.95 & $3.871(5)$ & 160 \\
$\mathrm{C} 13-\mathrm{H} 13 B \cdots C g 9$ & 0.96 & 2.92 & $3.680(9)$ & 137
\end{tabular}

Symmetry codes: (i) $-x+2, y+1 / 2,-z+1$; (ii) $-x+2, y-1 / 2,-z$; (iii) $x, y, z-1$; (iv) $-x+2, y+1 / 2,-z$; (v) $x, y, z+1$; (vi) $-x+2, y-1 / 2,-z+1$; (vii) $x-1, y, z$. 